\title{
Política y realidad: un análisis sobre la evaluación interna en lengua castellana ${ }^{1}$
}

Mónica González

monicavgonzalezc@gmail.com

Bibiana Pinto ${ }^{2}$

bibi_pm7@hotmail.com

REsUMEN

Este estudio se ocupa de la evaluación interna en el área de Lengua Castellana como proceso mediado por las políticas del Ministerio de Educación Nacional. Surge de la necesidad de establecer la relación entre la intencionalidad expresada en las normas y su efectiva incidencia en las prácticas de aula, para lo cual recurrió a un estudio de caso de carácter etnográfico en la Institución Educativa oeA realizado durante el año 2005.

\section{Palabras clave}

Evaluación interna, lengua castellana, política educativa, prácticas de aula.

\section{Abstract}

This study analyses the internal evaluation in the area of Castilian Language as a process mediated by the policies or the Ministry of National Education. It arises from the necessity of establishing the relation between the intentionality expressed in the norms and their effective incidence in the classroom practices, for which it resorted to a case study of ethnographic character in the Educative Institution OEA carried out during 2005.

\section{KEYWORDS}

Internal evaluation, Castilian language, educative policies, classroom practices.

1 Este artículo presenta los resultados finales de la monografía de grado titulada Política y realidad: un análisis sobre la evaluación interna en Lengua Castellana, realizado por los miembros del Semillero de Investigadores del grupo de investigación "Lenguaje, Identidad y Cultura" de la Universidad Distrital Francisco José de Caldas, en el marco del Proyecto Interinstitucional "Constitución de una Red Nacional de Investigación en Evaluación (Convenio Universidad Nacional-Universidad Distrital con el aval de Colciencias 2005-2007).

2 Licenciadas en Educación Básica con énfasis en Lengua Castellana de la Universidad Distrital. Miembros del Semillero de Investigación en Evaluación del Grupo de Investigación Lenguaje Identidad y Cultura. 


\section{Introducción}

$\mathrm{L}$ a evaluación es un proceso inhierente a las prácticas académicas y todos los docentes la realizan constantemente para determinar los procesos de aprendizaje de sus educandos. Sin embargo se trata de un tema polémico, puesto que se presentan contradicciones entre las políticas del Estado y lo que efectivamente se realiza en el aula. Por una parte, en procura del mejoramiento de la calidad de la educación el Estado ha expedido nuevas normas sin tener en cuenta el necesario proceso de asimilación por parte de las diversas instancias del sistema; por otra, surge la inconformidad de algunos docentes frente a tales políticas, bien sea por desconocimiento respecto a su pertinencia, por su escasa apropiación y puesta en funcionamiento en las prácticas o por su incomprensión sobre la intencionalidad que las motiva.

Aunque siempre han existido problemas en el ámbito de la evaluación, éstos se han agudizado por los cambios introducidos a partir del año 2001 con la derogación de la Resolución 2324 relacionada con los Indicadores de Logros establecidos en la Ley 715, la introducción de la evaluación por competencias en los Exámenes de Estado y la expedición del Decreto 0230 y los Estándares en el 2002 y 2003, cuando aún los lineamientos curriculares expedidos en 1998 no se conocían suficientemente y su implementación era incipiente.

Frente a este problema, se asumió como propósito en la monografía que fundamenta el presente artículo conocer el grado de apropiación logrado por los docentes sobre las políticas educativas referentes a la evaluación y su incidencia en las prácticas de aula. El trabajo se propuso analizar las políticas educativas sobre la evaluación en Lengua Castellana; observar los modos de evaluación en el aula, las estrategias utilizadas y los contenidos evaluados, y determinar la interpretación de los docentes respecto a las políticas de evaluación en el aula de Lengua Castellana. En otras palabras, establecer la relación existente entre política y realidad a partir de la evaluación interna.

\section{Aspectos metodológicos del estudio}

Este estudio de caso optó por la investigación etnográfica (Rockwell, 1985 y Martínez, 1997), por considerarla una alternativa metodológica adecuada para la observación, la interpretación y el análisis de escenarios culturales naturales, a fin de comprender una situación y emprender procesos de transformación. Con el fin de evidenciar la puesta en práctica de las políticas sobre evaluación se seleccionó la Institución Educativa Distrital OEA, en la que se desarrolló un trabajo de campo con una duración de seis meses. Como lo señalan Rockwell y Mercado (2005), las condiciones materiales, los saberes de los maestros y la historia de la institución permitieron develar el proceso académico y sociocultural en el que estaban inmersos estudiantes y maestros.

Las observaciones de aula se llevaron a cabo en dos grupos: uno del grado $4^{\circ}$ de básica primaria y otro de $7^{\circ}$ de básica secundaria, cada uno con cuarenta estudiantes aproximadamente. Se utilizaron los diarios de campo como herramienta para detectar problemas y hacer explícitas las concepciones de los docentes sobre la evaluación, permitiendo la valoración de las prácticas docentes; para conocer su interpretación sobre las políticas se realizaron entrevistas semiestructuradas o focales dirigidas a profesores de Lengua Castellana y a coordinadores.

\section{Análisis de resultados}

A continuación se plantean los principales aspectos relacionados con la evaluación interna en Lengua Castellana, lo cual permite contrastar qué se evalúa y cómo se evalúa frente a qué piensan los docentes respecto a sus prácticas de evaluación.

Los registros de los diarios de campo permitieron comprender de qué modo se orientan en el aula las actividades del lenguaje propuestas 
en los lineamientos y los estándares curriculares y establecer qué se evalúa y qué estrategias se emplean con tal propósito. De estas observaciones se concluye que pese a los cambios operados en las políticas, en el aula predominan las formas de evaluación tradicional, caracterizadas de acuerdo con Bustamante (1999) como repetitivas en cuanto se asume que evaluar consiste en confrontar objetivos y resultados, lo que implica que objeto y sujeto se enfrenten, con la mediación de un método que garantice su confiabilidad.

En cuanto a la lectura la evaluación se sitúa por lo general en el nivel literal y busca esencialmente establecer la correspondencia entre lo identificado en el texto y las respuestas solicitadas en los cuestionarios formulados o las pautas dadas por los docentes. Así, en el caso de $4^{\circ}$ grado de básica primaria, para cada periodo está programado que los estudiantes lean un libro y al final hagan entrega de un análisis literario, que consta de ocho purtos: argumento de la obra, personajes principales y secundarios, tiempo, espacio, lenguaje utilizado, narrador, partes y autor. Este análisis se valora usando la escala evaluativa propuesta por el Decreto 0230 de 2002. Respecto a la lectura en voz alta se tienen en cuenta aspectos como las pausas y la entonación, con total predominio de lo fónico y limitada atención a los procesos de comprensión lectora. Habitualmente se realizan algunas preguntas literales con el fin de mantener el orden y controlar la atención. A través de lo oral los docentes valoran la actitud de -los estudiantes frente a la convivencia y las actividades de aprendizaje propuestas, aunque también los evalúan por escrito para que quede un reporte de la valoración.

En cuanto a la escritura se privilegian los aspectos gráficos, insistiendo en que las grafías sean legibles, por lo cual se les exige a los estudiantes la letra técnica, que consiste en que la minúscula ocupe un cuadro de la hoja (en cuaderno cuadriculado) y la-mayúscula dos cuadros de alto y uno y medio de ancho. Para reforzar esta actividad y velar por la corrección ortográfica habitualmente a los estudiantes se les entrega un libro del cual deben copiar algunos textos éscogidos por el docente con anticipación y repetir los ejercicios cuantas veces sea necesario hasta cuando la letra quede según las instrucciones.
Esto conlleva a una nota y es la razón por la cual la mayor preocupación de los estudiantes es que "les quede bonito todo lo que hacen".

Tanto en $4^{\circ}$ grado como en $7^{\circ}$, la escritura es una actividad constante en el desarrollo de las clases y se utilizan estrategias de evaluación como tareas, talleres, copias y dictados, y se dejan tareas para reforzar el tema estudiado. Se puede decir que este tipo de actividad se convierte en una estrategia esencial de evaluación para el docente. La realización escrita de talleres se presenta como una actividad en clase. Éstos, por lo general, son extensos, ya que constan de entre 16 y 20 puntos extraídos de libros de texto, por lo que en algunas ocasiones poseen terminología difícil que tampoco es explicada por el docente. Aunque algunos estudiantes se arriesgan a preguntar, la mayoría lee y copia en sus cuadernos sin entender lo que se está escribiendo. Esto se hace evidente sólo en el momento de la evaluación de dichos talleres, ya que los estudiantes no responden las preguntas: dejan los espacios en blanco y obtienen una nota deficiente. En cuanto a las estrategias empleadas para evaluar contenidos se realizan pruebas escritas, como quizes, que permiten ver qué tanto aprendieron sobre determinado tema; así mismo se les solicita a los estudiantes que preparen exposiciones en grupo para ser evaluadas.

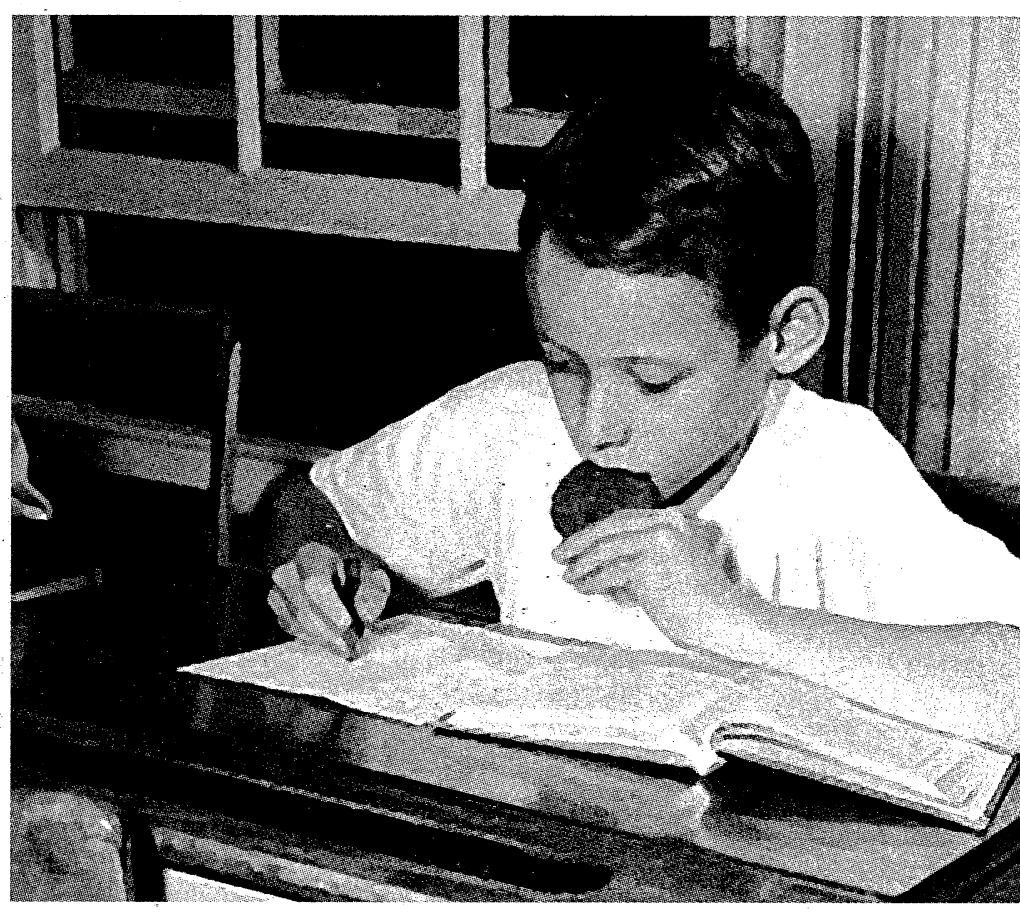


Tanto en básica primaria como en básica secundaria se tienen en cuenta los criterios de evaluación definidos por la institución y los establecidos por el Decreto 0230 de 2002, y se recurre a la valoración conceptual expresada en: Excelente (E), Sobresaliente (S), Aceptable (A), Insuficiente (I) y Deficiente (D), según el estudiante cumpla o no con lo solicitado. Para obtener el resultado de la evaluación por lo general se suma la cantidad de errores, así: ningún error $(E)$, uno o dos errores $(\mathrm{S})$, tres o cuatro errores $(\mathrm{A})$, cinco errores en adelante (I) y si no la presenta (D). Estas valoraciones se consignan en el trabajo realizado por el estudiante y algunas de ellas se pasan a las planillas de evaluación de la institución, al lado de una valoración cualitativa que muestra los logros, las dificultades y las fortalezas que evidencia el estudiante.

Las entrevistas realizadas permitieron identificar los paradigmas en los cuales se ubican las concepciones de los maestros frente a la evaluación. De este análisis se concluye que la mayoría de ellos se sitúa en el paradigma tradicional, una mínima parte en el paradigma por procesos mientras que una franja intermedia transita entre las dos concepciones.

En efecto, para el 53\% de los docentes ubicados en el paradigma tradicional la evaluación busca "fijar el conocimiento, ver cuánto comprendió [el estudiante]" o "medir resultados". Por otra parte, el 13\% de los docentes entrevistados dice asumir una evaluación por procesos, paradigma según el cual, de acuerdo con Bustamante (1993), evaluar no implica que el sujeto esté frente al objeto para aprehenderlo, ya que éste es un sujeto en proceso y, por ende, ninguno de los dos se encuentra determinado. Esta tendencia se centra en el estudiante y en los procesos de construcción de conocimiento basados en el aprendizaje. La prioridad se sitúa en la forma como aprenden los estudiantes y en sus procesos de conocimiento, y no simplemente en los resultados que alcanzan en las pruebas, lo cual implica la realización de un seguimiento permanente al desarrollo académico de cada uno. Los docentes situados en este paradigma plantean que se proponen "cuantificar los procesos que tienen los niños del aprendizaje", generando una reflexión sobre los avances cognitivos del estudiante, entendida la evaluación como una "metodología y diversidad de actividades para analizar un proceso". De esta forma, la evaluación cobra sentido en el aula, para "mirar al estudiante de acuerdo a como ha aprendido durante cada periodo y sus procesos. Evaluarlo de acuerdo a sus conocimientos [...]" y se constituye

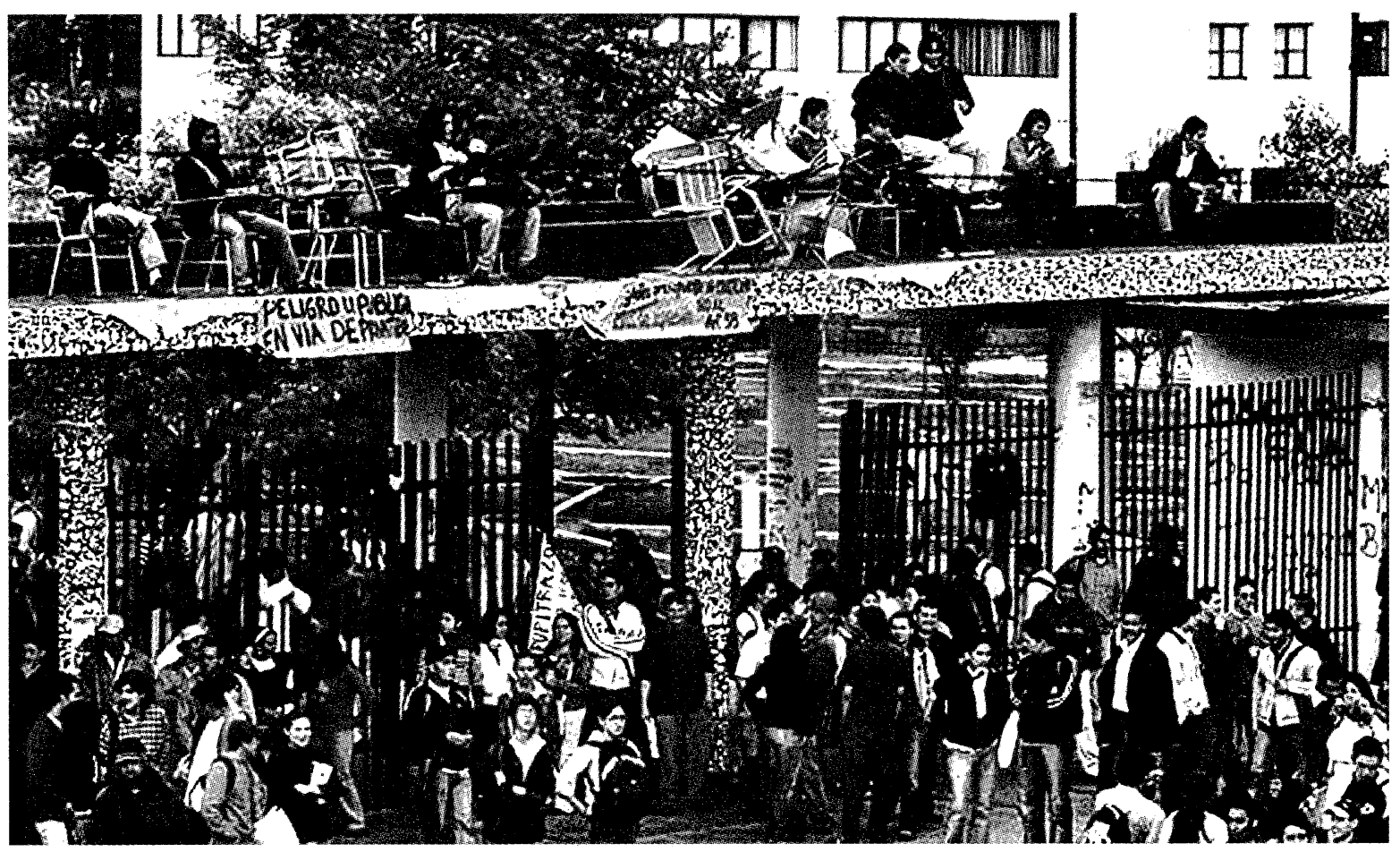


en punto de partida para mejorar los procesos de construcción de conocimiento.

Finalmente, en un punto intermedio entre la evaluación tradicional y la evaluación por procesos se ubica el 33\% de los docentes entrevistados, que demuestran la falta de apropiación frente a alguna concepción específica. Estos utilizan el término proceso para mostrar un recorrido de medición y no la evolución de cada estudiante en la construcción del conocimiento, siendo la evaluación un "proceso para medir conocimientos y rendimiento escolar".

\section{La observación de las prácticas de evaluación interna y la manera como los docentes se apropian de las políticas señalan que se requiere un constante estudio, análisis y comparación entre la imposición de la norma, su comprensión y su implementación, ya que los resultados muestran la gran distancia existente entre la política y la realidad.}

En cuanto a la apropiación lograda por los docentes frente a los lineamientos curriculares en Lengua Castellana (1998), se evidenció que se entienden como la formulación de parámetros en las áreas obligatorias, reglamentados por la Ley General de Educación (1994): "es una de las normas que uno debe conocer". Aunque en algunos docentes no hay claridad frente a quién los expide, si la Secretaria de Educación o el MEN. Esto se evidencia en afirmaciones como "todo va de acuerdo con los lineamientos que presenta la Secretaría de Educación" o "los lineamientos son como unos instrumentos, unos documentos que sacó el Ministerio de Educación ya hace algunos años".

Así mismo, los docentes utilizan los lineamientos como una guía que orienta la formulación del programa curricular: con ellos se proponen los logros y las competencias que el educando debe alcanzar, "se usan para estar acorde con lo reglamentado por la ley y la institución”. Por esta razón, los lineamientos se han asumido como pauta que marca parámetros al docente al momento de evaluar. Sin embargo son pocos los maestros que hacen un uso conciente de ellos. Al parecer no hay la suficiente apropiación de los ejes propuestos para poderlos llevar a la práctica. Así, un docente considera que "los lineamientos curriculares son las dimensiones, [que] aquí son las áreas obligatorias".

$\mathrm{Al}$ indagar sobre cuánto conocen los maestros acerca del Decreto 0230 de 2002, se encontró que para la mayoría se trata de un documento legal que debe ser aplicado, guste o no, por el simple hecho de que la norma implica "publíquese, comuníquese y cúmplase". Entendido como norma, este decreto ha sido uno de los que más polémica ha generado en el ámbito educativo, y se pueden identificar posturas a favor o en contra. Para las primeras, se trata de un documento bien elaborado, que permite una comprensión de la propuesta política en materia de currículo, promoción de los educandos y evaluación institucional y afirman que "el decreto orienta la forma de evaluar en las instituciones, da las pautas cualitativas para hacerlo y que sean de manera integral" y a la vez "es la herramienta de la cual el maestro debe valerse para poder hacer un buen proceso de evaluación".

Sin embargo, predominan las críticas al decreto, ya que buena parte de los docentes valora negativamente la propuesta de evaluación, de promoción automática y de trabajos de refuerzo que se deben realizar con los estudiantes que no logren alcanzar los objetivos propuestos por el plan de estudio. La mayoría de los docentes concuerda en que "lo único en que no estamos de acuerdo es en la promoción porque eso de que no pierdan los niños el año, ¡no!", o bien afirman: "yo le veo una falla sobre la evaluación y promoción de los educandos porque según ese decreto debe quedarse un número ínfimo de niños, cuando en realidad varios no han cumplido con los logros propuestos". De allí que consideren que se pierde la autonomía en el aula al evaluar en cuanto están obligados a promover estudiantes que según su criterio no se encuentran bien preparados para avanzar al siguiente curso. Esta opinión es la más fuerte y ratifica su inconformidad frente a esta propuesta que, a su juicio, afecta la calidad de la educación. 
Frente a los Estándares Básicos de Calidad de Lenguaje (2003), se evidenció que son asumidos como una forma para promover y desarrollar las cuatro habilidades básicas del lenguaje y al mismo tiempo como norma que se implementa en la institución. Según algunos entrevistados estos referentes normativos constituyen una forma de organización académica que les permite trabajar las cuatro habilidades básicas: leer, escribir, hablar y escuchar. Resaltan al respecto el énfasis otorgado a la producción de textos en cuanto al aspecto gramatical y las actividades que permiten hacer uso de la imaginación.

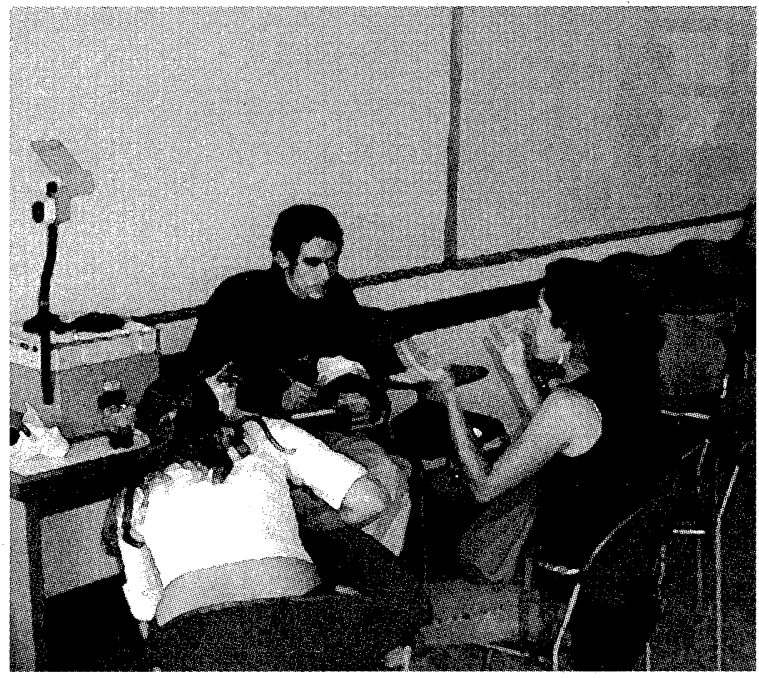

En síntesis, se concluye que los docentes conocen un poco más sobre la propuesta de los Estándares Básicos de Calidad de Lenguaje que sobre los lineamientos curriculares de Lengua Castellana porque hablan con mayor propiedad de los primeros, así dicho conocimiento no se vea reflejado en sus prácticas.

Las estrategias de evaluación son entendidas esencialmente como instrumentos que permiten observar qué tanto han aprendido los estudiantes, y la totalidad de los docentes coinciden en el uso de tareas extraclase que refuezan las actividades en clase desarrolladas de manera individual, grupal o lúdica, de acuerdo a la temática abordada.

Los ejercicios en clase consisten, la mayoría de las veces, en cuestionarios y talleres de selección múltiple, complementación, falso o verdadero y apareamiento a través de los cuales reconocen el grado de apropiación logrado por los educandos frente a los temas vistos. Las evaluaciones y los quizes responden a la necesidad de hacer explícitos los aprendizajes específicos sobre un tema. Generalmente se evalúa, según un docente, "a partir de los contenidos primero y a partir del grado”. Por lo tanto, las evaluaciones les permiten realizar un diagnóstico sobre la forma como los estudiantes construyen el conocimiento.

Según lo dicho por los docentes, la participación en clase y las actividades de refuerzo son herramientas utilizadas para promover el desarrollo de competencias en los estudiantes, haciendo de la evaluación un proceso integral que se preocupa por la enseñanza y el aprendizaje de cada estudiante, velando por la calidad de la educación y particularmente por dar cumplimiento al Decreto 0230 de 2002, que propone las actividades de refuerzo como una forma de ayuda a los estudiantes que se encuentran con bajo rendimiento académico.

\section{Conclusiones}

El análisis de las políticas de evaluación indican que éstas se conciben como una obligación del Estado para garantizar la calidad de la educación como derecho fundamental de todo ciudadano colombiano y la formación de ciudadanos competentes. Sin embargo, la observación de las prácticas de evaluación interna y la manera como los docentes se apropian de las políticas señalan que se requiere un constante estudio, análisis y comparación entre la imposición de la norma, su comprensión y su implementación, ya que los resultados muestran la gran distancia existente entre la política y la realidad.

Aunque la evaluación se haya constituido en un referente indispensable para promover la educación de calidad, se requiere profundizar la reflexión sobre los fundamentos teóricos y sus nexos con las prácticas efectivas en el aula a fin de buscar estrategias de mejoramiento a partir de las cuales se logre una mayor incidencia en la formación integral de los estudiantes.

Efectivamente, el análisis de las observaciones y las entrevistas realizadas en este estudio muestran que las prácticas evaluativas siguen ancladas en el paradigma tradicional, por cuanto la calidad promulgada por las políticas 
del MEN no se evidencia en la realidad escolar, debido, entre otras razones, a que las concepciones de los docentes están alejadas de los planteamientos teóricos que subyacen a làs normas. Por consiguiente, el Estado debería preocuparse de por qué los docentes en general no conocen debidamente su propuesta y a través de qué acciones se pueden actualizar las concepciones de los docentes.

Las estrategias utilizadas para la evaluación en el aula son las tradicionales y a través de ellas se espera que todos los estudiantes respondan de manera homogénea, de forma que muy poca incidencia han tenido las políticas que promueven una evaluación por procesos. Esta situación anula las posibilidades de innovación y desconoce la participación e intereses de los estudiantes en sus procesos de aprendizaje.

Finalmente es necesario señalar que la evaluación en Lengua Castellana se constituye como una herramienta que permite medir el nivel sobre cuanto saben los estudiantes por medio de la memorización, el cuaderno y las evaluaciones escritas, entre otras, sin lograr la consolidación de un proceso evaluativo que responda a una propuesta de calidad en el aula. En síntesis: las políticas de evaluación y las prácticas efectivas en torno a la evaluación interna son dos vías que no logran aún la integración necesaria. Ó

\section{Bibliografía}

Bustamante, G. (1999), La evaluación, Vida de maestro, Una pasión hecha proyecto, Bogotá, IDEP.

Casanova, M. A. (1999), "Evaluación y mejora de la calidad educativa. ¿Para qué evaluar?" en Educación y Cultura, núm. 49, Bogotá.

CEID-Fecode (2002), "La evaluación escolar y el decreto 230", en Educación y Cultura, núm. 61, Bogotá.

Colectivo Sociedad Colombiana de Pedagogía (2002), "Los estándares curriculares: Trivialización del acontecimiento pedagógico", en Educación y Cultura, núm. 6, Bogotá.

Constitución Política de Colombia 1991 (2005), Librería la Constitución.

Decreto 0230 (2002), República de Colombia.
Duarte, P. y Cuchimaque, E. (1999), Examen de Estado, cambios para el siglo XXI, Lenguaje", Bogotá, IcFes.

Evaluación de Competencias Báșicas en Bogotá (1998-2001), [CD-ROM].

Ferreiro, E. (1999), Cultura escrita y educación. Conversaciones con Emilia Ferreiro, México, FCE.

Guevara, C. et al. (2004), Tradición investigativa en le programa de lingüistica y literatura [documento de trabajo], Bogotá, Universidad Distrital Francisco José de Caldas.

Herrera, M. y Palacio, J. (s.f), Evaluación por logros [documento de trabajo], Bogotá, Universidad Pedagógica Nacional.

Jurado, F. et al. (2001), "La evaluación censal de competencias básicas en Bogotá", en Educación y Cultura, núm. 56, Bogotá.

Martínez, M. (1997), "La investigación cualitativa etnográfica en Educación" [manual teórico práctico capítulos 4 y 5], Bogotá, Círculo de Lectura Alternativa.

Ministerio de Educación Nacional (2003), "Estándares Básicos de Calidad de Lenguaje”, Bogotá.

Ministerio de Educación Nacional (1994), Ley General De Educación 115, Bogotá.

Ministerio de Educación Nacional (1998), Lineamientos Curriculares Lengua Castellana, Bogotá, Magisterio.

Montenegro, I. y Ayarza, A. (2003), "El camino de la evaluación" entrevista a Daniel Bogoya, en Educación y pedagogía, núm. 1, Bogotá, Magisterio.

Red colombiana para la transformación de la formación docente en Lenguaje (2003), "Frente a los estándares curriculares. $\mathrm{El}$ caso de Lengua Castellana y Literatura”, Bogotá, Magisterio.

Rockwell, E. (1985), "Etnografía y teoría en la investigación educativa en Tercer Seminario Nacional de Investigación en Educación", Bogotá, Universidad Pedagógica Nacional.

- y Mercado, R. (2005), "La práctica docente y la formación de maestros", en Modulo de práctica docente 1, Bogotá, Universidad Distrital Francisco José de Caldas. 
Rodriguez, I. (1999), La innovación educativa: una forma de descifrar la existencia pedagógica, Una pasión hecha proyecto, Bogotá, IDEP.

Rodríguez, R. H. (2000), La evaluación en el aula, elemento para identificar y replantear formas de enseñanza en la institución, Bogotá, Unidad Básica Rafael Uribe Uribe.

Sánchez Murillo, W. R. (2003), "Calidad de la educación: una oportunidad para la Ley General de Educación", en Educación y Cultura, núm. 64, Bogotá; Fecode.

Secretaría de Educación (2000), "Resultados evaluación de competencias básicas en Lenguaje y Matemáticas", Bogotá, Secretaría de Educación.

Secretaría de Educación (2004), "Evaluación censal de competencias en el grado obligatorio de preescolar: transición”, Bogotá, Secretaría de Educación.
Urrego, J. de D. (s.f.), Acerca de los paradigmas y las competencias, Bogotá, Santillana.

- y Urrego, H. (s.f.), Las competencias: una necesidad educativa para el nuevo siglo, Bogotá, Santillana.

Vargas, M. (1999), Aprender a aprender. Argumento de la innovación. Una pasión hecha proyecto, Bogotá, IDEP.

Vasco, C. E. (2003), "Objetivos específicos, indicadores de logro y competencias: ¿y ahora estándares?”, en Educación y Cultura, núm. 62, Bogotá, Fecode.

Vasco, M. E. (1999), Reflexiones sobre la innovación en la escuela: a propósito de algunas bistorias de maestros. Una pasión hecha proyecto, Bogotá, IDEP.

Zubiría, J. (s.f.), La universidad y el fracaso escolar. Una mirada desde la educación media [documento de trabajo]. 